\title{
Alternating Coupling Regimes in a Plasmon-Molecule Hybrid Structure through Phase-Change Material
}

\section{Supporting Information}

\author{
Yun-Wei Chang, Jie Yao, ${ }^{*}$ Xing-Feng Zhu, Qi Wei, and Da-Jian Wu* \\ Jiangsu Key Lab of Opto-Electronic Technology, School of Physics and Technology, \\ Nanjing Normal University, Nanjing 210023, China
}

\section{Corresponding authors:}

Jie Yao - Jiangsu Key Lab of Opto-Electronic Technology, School of Physics and Technology, Nanjing Normal University, Nanjing 210023, China; Email: yaojie1@njnu.edu.cn

Da-Jian Wu - Jiangsu Key Lab of Opto-Electronic Technology, School of Physics and Technology, Nanjing Normal University, Nanjing 210023, China; Email: wudajian@njnu.edu.cn 


\section{S1. The bare PMMA polymer vibrational spectrum}

The PMMA molecules has a strong vibrational mode at $\sim 5.77 \mu \mathrm{m}$, which is corresponding to the stretch vibration of the carbonyl $(\mathrm{C}=\mathrm{O})$ group. The absorption spectrum of the PMMA grating is shown in Fig. S1. There is an absorption band at $\sim 5.77 \mu \mathrm{m}$, according to the $\mathrm{C}=\mathrm{O}$ group.

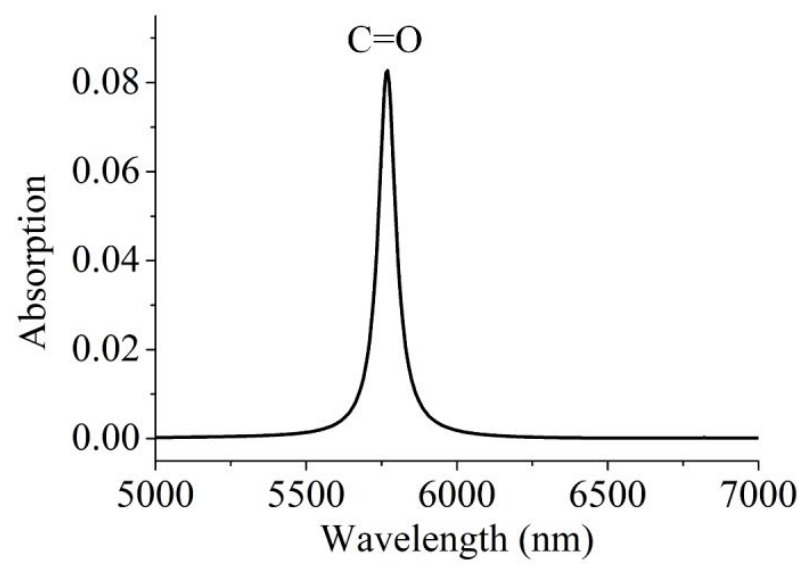

Figure S1. Absorption spectrum of the PMMA grating. 


\section{S2. Optical responses of the dielectric grating sitting on a bare gold substrate}

The optical response of the dielectric grating sitting on a bare gold substrate is calculated for comparison. The permittivity of the dielectric stripe grating was set as $\varepsilon$ $=\varepsilon_{\square}=2.36$ and the plot is shown in Fig. S2. Under normal incidence, the SPP mode is at around $5.88 \mu \mathrm{m}$. It splits into two branches with the increasing incident angle $\theta$.

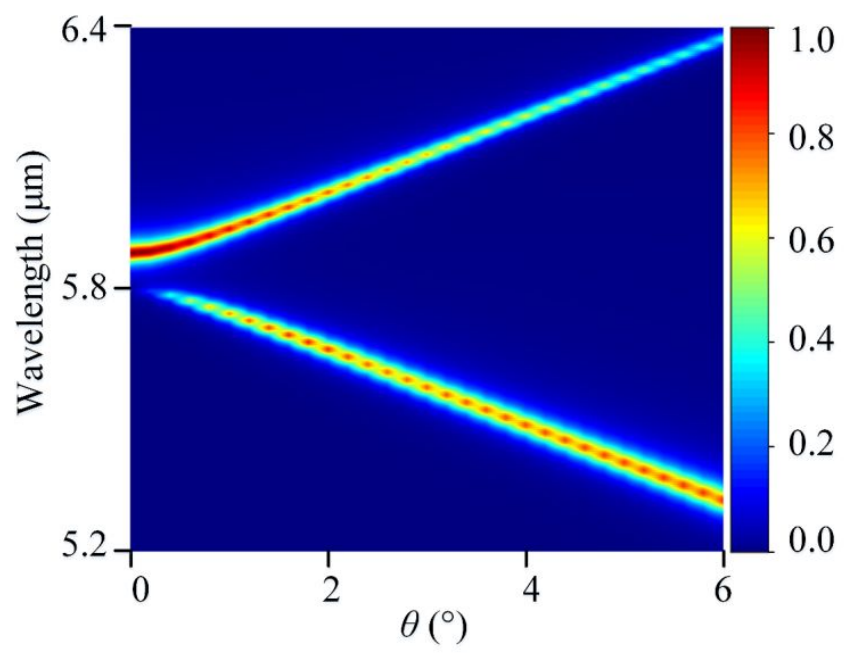

Figure S2. Contour plot of absorption for a dielectric grating sitting on the gold substrate. Here, $P=5500 \mathrm{~nm}, w=2500 \mathrm{~nm}$, and $h=800 \mathrm{~nm}$. 


\section{S3. Absorption spectra of different substrates}

When there are no stripe gratings, the absorption spectra of different substrates are shown in Fig. S3. The solid line stands for the absorption of the $\mathrm{VO}_{2}$ (insulator)- $\mathrm{Au}$ substrate and the dashed line refers to the $\mathrm{VO}_{2}$ (metal)- $\mathrm{Au}$ substrate.

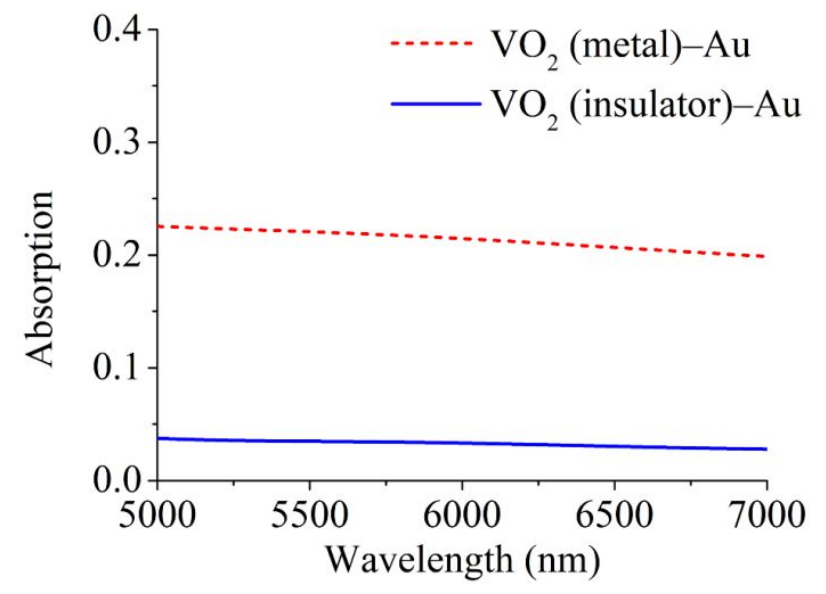

Figure S3. Absorption spectra of the $\mathrm{VO}_{2}$ (metal)- $\mathrm{Au}$ substrate and the $\mathrm{VO}_{2}$ (insulator)-Au substrate. 


\section{S4. Absorption of the PMMA-VO (insulator)-Au system under TE incidence}

Figure $\mathrm{S} 4$ shows the contour plot of the absorption spectra from the PMMA- $\mathrm{VO}_{2}$ (insulator)-Au structure under TE incidence. The structural parameters are the same as those in Fig.3. When the incident light is TE polarized, there is only the molecular vibration (MV) mode at $\sim 5.77 \mu \mathrm{m}$. The SPP cannot be excited and the anti-crossing behavior no longer exists accordingly.

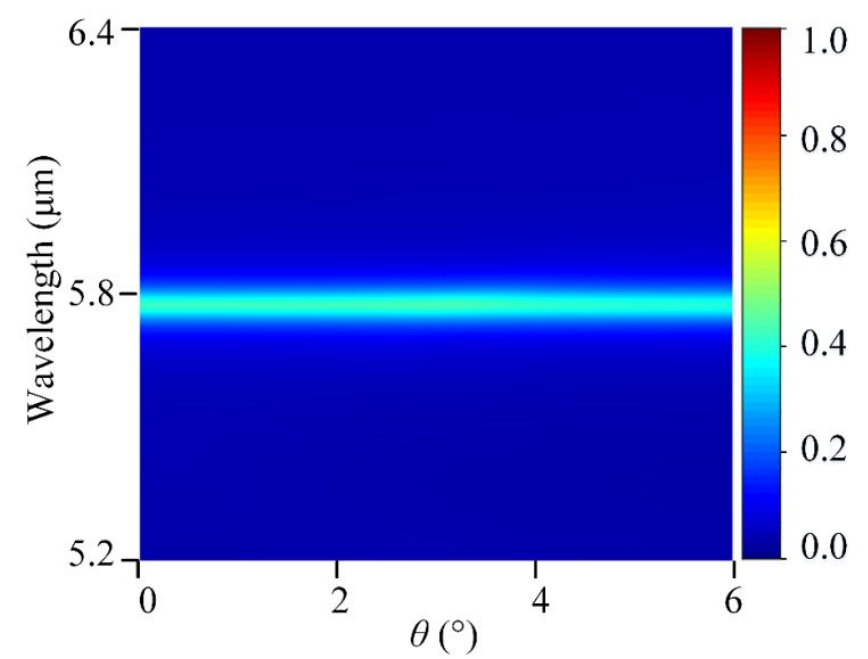

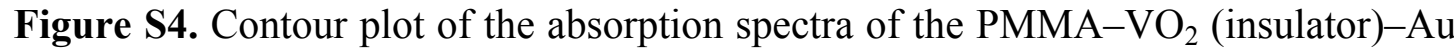
structure under TE incidence. Here, $P=5500 \mathrm{~nm}, w=2500 \mathrm{~nm}, h=800 \mathrm{~nm}$, and $t=$ $60 \mathrm{~nm}$. 


\section{S5. The red shifting SPP mode coupling with the MV mode}

The anti-crossing behavior can also exist between the red-shifting SPP mode and the MV mode when the grating period $P$ is smaller. For example, when $P$ is decreased to $4900 \mathrm{~nm}$, the SPP mode under normal incidence is near $\sim 5.60 \mu \mathrm{m}$, which is at the blue side of the MV mode $(5.77 \mu \mathrm{m})$. Thus the blue-shifting branch moves away from the MV mode while the red-shifting one approaches it with the increasing incident angle $\theta$ as shown in Fig. S5. When $\theta$ increases from $0^{\circ}$ to $6^{\circ}$, the red-shifting SPP mode moves across the MV mode. The strong coupling between the original modes results into the anti-crossing between two new hybrid polaritons. The dashed lines and the solid lines stand for the original modes and the hybrid polaritons respectively.

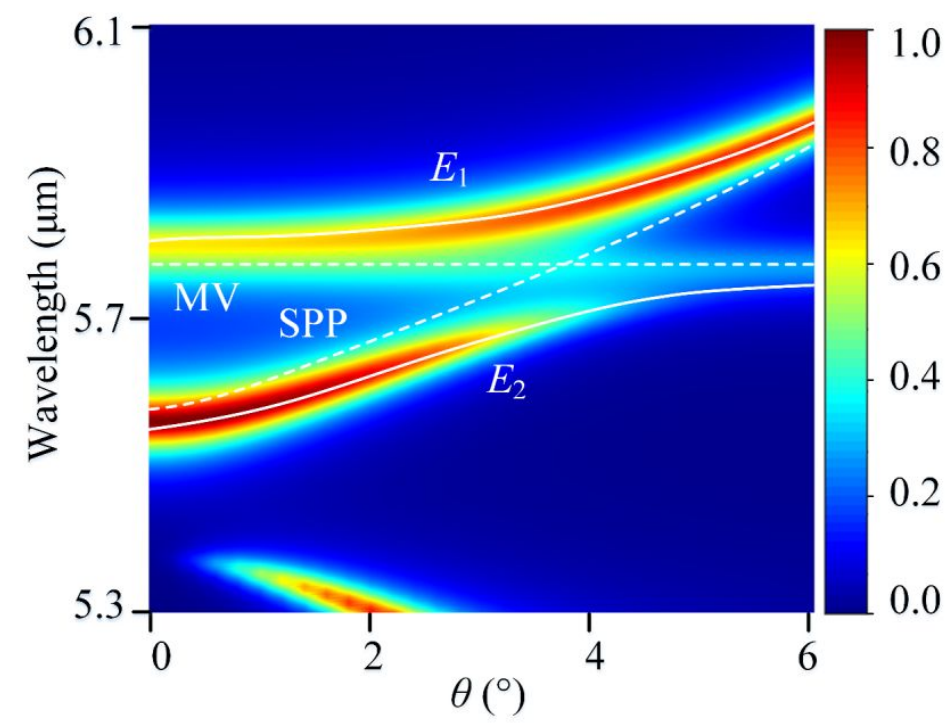

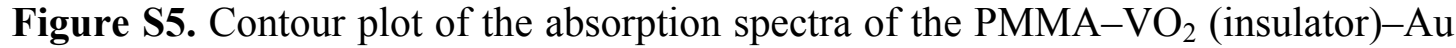
structure as a function of the incident angle $\theta$. Here, $P=4900 \mathrm{~nm}, w=2500 \mathrm{~nm}, h=$ $800 \mathrm{~nm}$, and $t=60 \mathrm{~nm}$. The dashed lines represent the original SPP and MV modes. The solid lines stand for the two new hybrid polaritons based on the oscillator model. 


\section{S6. The SPP mode coupling with the MV mode by adjusting the grating period}

The SPP mode depends on the grating period $P$ and the incident angle $\theta$. Thus coupling between the SPP and MV modes can also be investigated by varying the grating period. When $P$ increases from $4900 \mathrm{~nm}$ to $5500 \mathrm{~nm}$, the SPP mode excited under normal incidence moves from $\sim 5.60 \mu \mathrm{m}$ to $\sim 6.05 \mu \mathrm{m}$. It couples strongly with the MV mode and results the anti-crossing between two new hybrid polaritons as shown in Fig. S6.

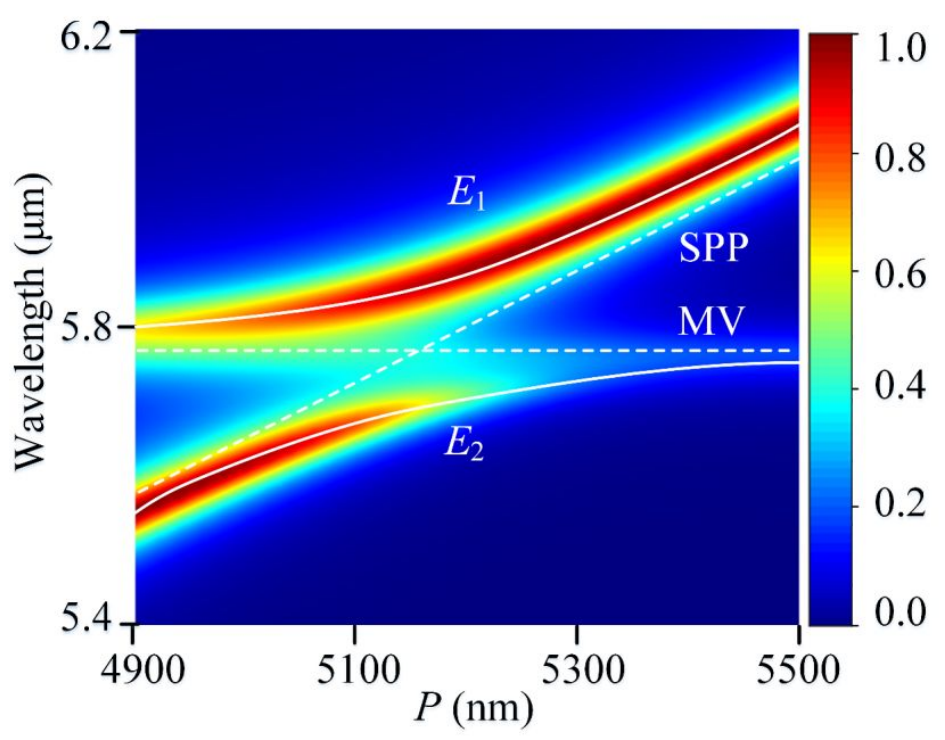

Figure S6. Contour plot of the absorption spectra of the coupled PMMA- $\mathrm{VO}_{2}$ (insulator)-Au structure as a function of the grating period $P$. Here, incident angle $\theta=$ $0^{\circ}, w=2500 \mathrm{~nm}, h=800 \mathrm{~nm}$, and $t=60 \mathrm{~nm}$. The dashed lines represent the original SPP and MV modes. The solid lines stand for the two new hybrid polaritons based on the oscillator model. 